\title{
Effect of age, sex and local government area on linear body measurements of WAD goat in Ogun State, Nigeria
}

Aluko, F. A., Daramola, O. E. and Taiwo, B. B. A.

Department of Animal Production, Olabisi Onabanjo University, P. M. B. 0012, Ayetoro, Ogun State.

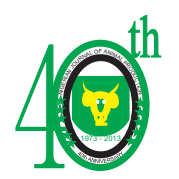

\begin{abstract}
One hundred and twenty West African Dwarf goats were used in this study. Observations were made from twelve different towns in Yewa North, Yewa South and Abeokuta North Local Government. West African dwarf goats were evaluated for phenotypic variation at different ages ranges from 1, 2 and 3 years. Variations in live weight and body measurements such as height at wither; body length, heart girth, fore limb length, hind limb length, face length, ear length, ear width, hip width and tail length were taken. The local government areas significantly $(P<0.05,0.001)$ affected body length, fore limb length, ear length, ear width, hip width and live weight. Age and sex significantly $(P<0.01)$ affected all the body measurements. The mean live weight by age groups are $6.61 \pm 0.31 \mathrm{~kg}, 13.10 \pm 0.60 \mathrm{~kg}$ and $20.18 \pm 0.95 \mathrm{~kg}$ for ages 1,2 and 3 respectively. The coat colour varied from black, brown, grey to white in WAD goats. Some colours were in patches, some uniform while others were spotted.
\end{abstract}

\section{Introduction}

Geneticists consider variation as the raw material for improvement; variation that is both visible (phenotypic) and invisible (genetic) and the breeder's tool to work on this variation is selection (Dalton, 1985). Campbell et al (2003) stated that genes possess the remarkable property of duplicating themselves precisely from generation to generation. Dalton (1995) further explained that the easiest way to understand variation in a flock or herd is to draw a diagram of it (called a distribution) for a particular trait using examples such as live weight at specific age, fleece weight, milk yield in a group of animal. Qualitative inheritance involves genetic principle that deals specifically with the individual and this is because there are distinct differences between phenotypes and because few genes are involved while quantitative inheritance involves the individual less and the total population more (Campbell et al, 2003). Linear body measurements provide good information on performance, productivity and carcass characteristics of animals (Ige et al, 2006). A study of linear body measurements on most farms in the tropics is important because most farmers lack weighing scales and the education to understand their manipulations (Gerald, 1994). Linear body measurement can be used as a way of estimating weight and market value in terms of cost of the animals (Gerald, 1994). The relationships existing among linear body traits provide useful information on performance, productivity and carcass characteristics; body size and shape (conformation) are important traits in meat animals. (Adewunmi et al, 2008). External body measurement of animal has been extensively used to assess the growth of skeletal parts; they are also useful in describing in a general way, changes in 
animal conformation with age (Ngere et al, 1984). The body weight of the live animal is the most comprehensive measure of growth generally available; but it's particularly subject to short term changes and receives the full impact of pregnancy and kidding (Adewunmi and Badepade, 2008).

\section{Materials and methods}

In this study, the local government areas covered were Yewa North, Yewa South and Abeokuta North. Four (4) towns were selected in each Local Government based on random selection. The experimental animals were WAD goats of different coat colour patterns. The goats were reared under the extensive system of management. A total of 120 animals were sampled at the rate of 10 animals per town giving a total of 40 animals per Local Government Area. Body weights were taken in the morning before the animals were fed using bathroom scale. All body measurements were taken using measuring tape either in the morning or late in the evening when indoor to prevent restless behaviours. The body parameters measured included, live weight (WT), height at wither (HTW), body length (BL), heart girth (HG), fore limb length (FL), hind limb length (HL), face length (FAL), hip width (HW), tail length (TL), ear length (ELN), ear width (EWD), age was determined using dentition as describe by Banerjee (2006). Each animal due for measurement was restrained. The experiment spanned 4-5 months from late December to late April 2010.

Analysis of variance was run to determine the effects of local government area, town, age and sex of animal on body weights and body measurements. The means were tested for significant differences by Duncan multiple-range test. Correlations were run between body measurements and body weights.

\section{Results and Discussion}

Local government areas significantly $(\mathrm{P}<0.05,0.01)$ affected body length, fore limb length, ear length, ear width, hip width and live weight. The town significantly $(\mathrm{P}<0.01,0.05)$ affected heart girth, face length and ear width. Age and sex significantly $(\mathrm{P}<0.01)$ affected all the body measurements. (Table 1).

WAD goats from Yewa South were significantly $(\mathrm{P}<0.05)$ higher than WAD goats from Yewa North and Abeokuta North in body length, fore limb length, ear length and live weight with the respective means $47.35 \pm 1.58 \mathrm{~cm}, 26.03 \pm 0.62 \mathrm{~cm}$, $9.56 \pm 0.22 \mathrm{~cm}$ and $14.45 \pm 1.15 \mathrm{~kg}$ (Table 2). The variations in WAD goat linear body measurements in the different local government areas could be as a result of the environmental influence such as climatic condition of the local government areas and the type of management system used on animals. WAD goats of age three (3) years was significantly $(\mathrm{P}<0.05)$ higher than WAD goats aged one (1) and two (2) years with respect to all body parameters taken. (Table 3). Table 3 shows the difference in mean live weight of WAD goats by age groups are $6.61 \pm 0.31 \mathrm{~kg}, 13.10 \pm 0.60 \mathrm{~kg}$ and $20.18 \pm 0.95 \mathrm{~kg}$ for ages 1,2 , and 3 respectively. These differences in body weights and body lengths of WAD goats at any age were highly significant at $\mathrm{P}<0.05$ (Table 1), because as the age increased, the live weight and size increased which therefore agreed with Nwangu (1982), who reported that there are potential for high rate of growth in goats. These results were in agreement with earlier report of McDonald et al (1981) who reported that various parts of the body grow at different rate, so that its proportion changes as the animal matures. The mean body length of WAD goats by age 
Aluko, Daramola and Taiwo

TABLE 1: Analysis of Variance on Body Parameters of WAD goats sampled

\begin{tabular}{|c|c|c|c|c|c|c|c|c|c|c|}
\hline \multicolumn{11}{|c|}{ MEAN SQUARES } \\
\hline & $\mathrm{BL}$ & $\mathrm{HG}$ & FL & HL & FAL & ELN & EWD & HW & TL & WT \\
\hline & $327.53 * *$ & 292.23 & $32.23 * * *$ & 22.16 & 11.10 & $4.77 * * *$ & $0.60 * * *$ & 23.70 & 3.80 & $156.17 * *$ \\
\hline & 128.02 & $366.51 * *$ & 15.52 & 20.60 & $11.84 * *$ & 2.42 & $0.60^{*}$ & 14.82 & 3.52 & 55.42 \\
\hline * & $3977.51 *$ & $6956.97 *$ & $468.45^{*}$ & $608.26^{*}$ & 198.09* & $50.85^{*}$ & $4.78^{*}$ & $\begin{array}{l}369.03 \\
*\end{array}$ & $87.85^{*}$ & $1890.47^{*}$ \\
\hline & $1367.33 *$ & $2170.71 *$ & $106.84 *$ & $208.16^{*}$ & $62.48^{*}$ & $6.65^{*}$ & $2.29 *$ & $\begin{array}{l}53.80 * \\
*\end{array}$ & $35.05^{*}$ & $491.17 *$ \\
\hline
\end{tabular}

\begin{tabular}{|c|c|c|c|c|c|c|}
\hline \multirow[t]{14}{*}{$\mathrm{P}<0.01 *$} & & $\mathrm{P}<0.05^{* *}$ & $\mathrm{P}<0.001 * * *$ & & & \\
\hline & \multirow{13}{*}{$\begin{array}{l}\mathrm{HTW}= \\
\mathrm{BL}= \\
\mathrm{HG}= \\
\mathrm{FL}= \\
\mathrm{HL}= \\
\mathrm{FAL}= \\
\mathrm{ELN}= \\
\mathrm{EWD}= \\
\mathrm{HW}= \\
\mathrm{TL}= \\
\mathrm{WT}=\end{array}$} & \multirow{13}{*}{$\begin{array}{l}\text { Height at Wither } \\
\text { Body Length } \\
\text { Heart Girth } \\
\text { Fore Limb Length } \\
\text { Hind Limb Length } \\
\text { Face Length } \\
\text { Ear Length } \\
\text { Ear Width } \\
\text { Hip Width } \\
\text { Tail Length } \\
\text { Body Weight }\end{array}$} & \multicolumn{4}{|c|}{$\begin{array}{l}\text { TABLE 2: Means of body measurements }(\mathrm{cm}) \text { and standard error (SE) of WAD goat } \\
\text { Local Government Areas of Ogun State. }\end{array}$} \\
\hline & & & L.G.A/Variables (cm) & $\begin{array}{c}\text { Yewa North } \\
\mathbf{N}=\mathbf{4 0}\end{array}$ & $\begin{array}{l}\text { Yewa South } \\
\quad \mathbf{N}=40\end{array}$ & $\begin{array}{c}\text { Abeokuta North } \\
\mathbf{N}=\mathbf{4 0}\end{array}$ \\
\hline & & & Height at Wither & $39.02 \pm 0.97^{\mathrm{a}}$ & $40.24+1.12^{\mathrm{a}}$ & $37.34 \pm 0.97^{\mathrm{a}}$ \\
\hline & & & Body Length & $42.27 \pm 1.47^{\mathrm{a}}$ & $47.35+1.58^{\mathrm{b}}$ & $42.52 \pm 1.41^{\mathrm{a}}$ \\
\hline & & & Heart Girth & $56.02 \pm 2.65^{\mathrm{a}}$ & $57.21 \pm 2.26^{\mathrm{a}}$ & $52.05+1.88^{\mathrm{a}}$ \\
\hline & & & Fore Limb Length & $25.02+0.58^{\mathrm{a}}$ & $26.03+0.62^{\mathrm{b}}$ & $24.25+0.54^{\mathrm{a}}$ \\
\hline & & & Hind Limb Length & $30.15+0.70^{\mathrm{a}}$ & $31.19+0.69^{\mathrm{a}}$ & $29.74+0.62^{\mathrm{a}}$ \\
\hline & & & Face Length & $14.31+0.41^{\mathrm{a}}$ & $15.01+0.42^{\mathrm{a}}$ & $13.98+0.37^{\mathrm{a}}$ \\
\hline & & & Ear Length & $8.92+0.22^{\mathrm{a}}$ & $9.56 \pm 0.22^{\mathrm{b}}$ & $9.02 \pm 0.17^{\mathrm{a}}$ \\
\hline & & & Ear Width & $3.14+0.09^{\mathrm{a}}$ & $3.18+0.05^{\mathrm{a}}$ & $2.95+0.09^{\mathrm{a}}$ \\
\hline & & & Hip Width & $13.03 \pm 0.59^{\mathrm{a}}$ & $14.22 \pm 0.52^{\mathrm{a}}$ & $12.77 \pm 0.50^{\mathrm{a}}$ \\
\hline & & & Tail Length & $8.58+0.31^{\mathrm{a}}$ & $8.49+0.25^{\mathrm{a}}$ & $8.01+0.23^{\mathrm{a}}$ \\
\hline & & & Body Weight (Kg) & $11.84 \pm 1.16^{\mathrm{a}}$ & $14.45 \pm 1.15^{\mathrm{b}}$ & $10.53 \pm 0.92^{\mathrm{a}}$ \\
\hline
\end{tabular}


Effect of age, sex and local government area on linear body measurements of WAD goat

TABLE 3: Means of body measurements (cm) and standard error (SE) of WAD goats sampled by Age

\begin{tabular}{lccc}
\hline Age (years)/Variables (cm) & $\mathbf{1}$ & $\mathbf{2}$ & $\mathbf{3}$ \\
& $\mathbf{N}=\mathbf{5 1}$ & $\mathbf{N}=\mathbf{3 1}$ & $\mathbf{N}=\mathbf{3 8}$ \\
\hline Height at Wither & $33.33 \pm 0.69^{\mathrm{a}}$ & $40.56 \pm 0.54^{\mathrm{b}}$ & $44.91 \pm 0.62^{\mathrm{c}}$ \\
Body Length & $35.25 \pm 0.76^{\mathrm{a}}$ & $46.15 \pm 0.92^{\mathrm{b}}$ & $54.13 \pm 0.77^{\mathrm{c}}$ \\
Heart Girth & $43.83 \pm 1.15^{\mathrm{a}}$ & $56.53 \pm 1.37^{\mathrm{b}}$ & $69.03 \pm 2.07^{\mathrm{c}}$ \\
Fore Limb Length & $21.98 \pm 0.35^{\mathrm{a}}$ & $26.25 \pm 0.28^{\mathrm{b}}$ & $28.35 \pm 0.48^{\mathrm{c}}$ \\
Hind Limb Length & $26.71 \pm 0.38^{\mathrm{a}}$ & $32.22 \pm 0.34^{\mathrm{b}}$ & $33.73 \pm 0.57^{\mathrm{c}}$ \\
Face Length & $12.36 \pm 0.25^{\mathrm{a}}$ & $15.43 \pm 0.25^{\mathrm{b}}$ & $16.40 \pm 0.33^{\mathrm{c}}$ \\
Ear Length & $8.11 \pm 0.13^{\mathrm{a}}$ & $9.76 \pm 0.17^{\mathrm{b}}$ & $10.11 \pm 0.14^{\mathrm{b}}$ \\
Ear Width & $2.78 \pm 0.06^{\mathrm{a}}$ & $3.22 \pm 0.08^{\mathrm{b}}$ & $3.41 \pm 0.06^{\mathrm{c}}$ \\
Hip Width & $10.66 \pm 0.35^{\mathrm{a}}$ & $13.97 \pm 0.47^{\mathrm{b}}$ & $16.42 \pm 0.32^{\mathrm{c}}$ \\
Tail Length & $7.09 \pm 0.13^{\mathrm{a}}$ & $8.53 \pm 0.24^{\mathrm{b}}$ & $9.92 \pm 0.25^{\mathrm{c}}$ \\
Body Weight (Kg) & $6.61 \pm 0.31^{\mathrm{a}}$ & $13.10 \pm 0.60^{\mathrm{b}}$ & $20.18 \pm 0.95^{\mathrm{c}}$ \\
\hline Means with different superscript along the same row are significantly $(\mathrm{P}<0.05)$ different
\end{tabular}

Means with different superscript along the same row are significantly $(\mathrm{P}<0.05)$ different

groups are: $35.25 \pm 0.76 \mathrm{~cm}, 46.15 \pm$ $0.92 \mathrm{~cm}$ and $54.13 \pm 0.77 \mathrm{~cm}$ for ages $1, \overline{2}$, and 3 respectively Table 3 .

Table 4 shows that the female sex had higher values for all the body parameters measured than the males. It agrees with Fajemilehin and Salako, 2008 who reported that does were heavier than buck at the ages and the linear measurements were superior because female goat grows faster than the bucks at early stage. This was not similar to the findings Egena et al (2010) who stated that males were higher in body weight and body measurements than females except for length of ear and Ogah et al. (2013) who stated that sex affected most of the traits in favour of the male except for height at wither. This variation might be environmental differences and management.

Correlation between body weights and linear body measurements were high and

TABLE 4: Means of body measurements $(\mathrm{cm})$ and standard error (SE) of WAD goats sampled by sex

\begin{tabular}{lcc}
\hline Sex/Variables $(\mathbf{c m})$ & $\begin{array}{c}\text { Male } \\
\mathbf{N}=\mathbf{4 1}\end{array}$ & $\begin{array}{c}\text { Female } \\
\mathbf{N}=\mathbf{7 9}\end{array}$ \\
\hline Height at Wither & $36.03 \pm 1.04^{\mathrm{a}}$ & $40.34 \pm 0.67^{\mathrm{b}}$ \\
Body Length & $39.36 \pm 1.33^{\mathrm{a}}$ & $46.48 \pm 1.05^{\mathrm{b}}$ \\
Heart Girth & $49.19 \pm 1.82^{\mathrm{a}}$ & $58.15 \pm 1.69^{\mathrm{b}}$ \\
Fore Limb Length & $23.79 \pm 0.57^{\mathrm{a}}$ & $25.78 \pm 0.40^{\mathrm{b}}$ \\
Hind Limb Length & $28.53 \pm 0.59^{\mathrm{a}}$ & $31.31 \pm 0.47^{\mathrm{b}}$ \\
Face Length & $13.43 \pm 0.39^{\mathrm{a}}$ & $14.95 \pm 0.27^{\mathrm{b}}$ \\
Ear Length & $8.84 \pm 0.17^{\mathrm{a}}$ & $9.34 \pm 0.16^{\mathrm{a}}$ \\
Ear Width & $2.90 \pm 0.07^{\mathrm{a}}$ & $3.19 \pm 0.06^{\mathrm{b}}$ \\
Hip Width & $12.41 \pm 0.49^{\mathrm{a}}$ & $13.82 \pm 0.39^{\mathrm{a}}$ \\
Tail Length & $7.61 \pm 0.22^{\mathrm{a}}$ & $8.75 \pm 0.20^{\mathrm{b}}$ \\
Body Weight $(\mathrm{Kg})$ & $9.54 \pm 0.84^{\mathrm{a}}$ & $13.85 \pm 0.83^{\mathrm{b}}$ \\
\hline
\end{tabular}

Means with different superscript along the same row are significantly $(\mathrm{P}<0.05)$ different 
Aluko, Daramola and Taiwo

Table 5: Correlations among measured body parameters in WAD goats 1 year of age

\begin{tabular}{|c|c|c|c|c|c|c|c|c|c|c|c|}
\hline $\begin{array}{c}\text { Variable } \\
\mathrm{s}\end{array}$ & H W & BL & HG & FL & HL & FAL & ELN & EWD & HW & TL & WT \\
\hline HW & 1 & 0.799 ** & $0.755^{* *}$ & $0.835^{* *}$ & $0.726^{* *}$ & $0.735^{* *}$ & $0.437 * *$ & $0.638^{*}$ & $0.722 * *$ & $0.702^{* *}$ & $0.710^{* *}$ \\
\hline BL & $0.799 * *$ & 1 & $0839 * *$ & $0.683^{* *}$ & $0.549 * *$ & $0.642 * *$ & $0.551 * *$ & $0.545^{* *}$ & $0.680^{* *}$ & $0.550^{* *}$ & $0.741^{* *}$ \\
\hline HG & $0.755^{* *}$ & $0.839 * *$ & 1 & $0.683 * *$ & $0.570 * *$ & $0.667 * *$ & $0.588^{* *}$ & $0.551 * *$ & $0.715^{* *}$ & $0.535^{* *}$ & $0.735^{* *}$ \\
\hline FL & $0.835^{* *}$ & $0.683^{* *}$ & $0.683^{* *}$ & 1 & $0.841 * *$ & $0.880^{* *}$ & $0.435^{* *}$ & $0.714 * *$ & $0.698 * *$ & $0.676^{* *}$ & $0.781 * *$ \\
\hline HL & $0.726^{* *}$ & 0.549 ** & $0.570^{* *}$ & $0.841 * *$ & 1 & $0.800 * *$ & $0.467 * *$ & $0.679 * *$ & $0.564 * *$ & $0.548^{* *}$ & $0.626^{* * *}$ \\
\hline FAL & $0.735^{* *}$ & $0.642^{* *}$ & $0.667^{* *}$ & $0.880^{* *}$ & $0.800 * *$ & 1 & $0.508^{* *}$ & $0.590 * *$ & $0.650 * *$ & $0.616^{* *}$ & $0.623 * *$ \\
\hline ELN & $0.437^{* *}$ & $0.551^{* *}$ & $0.588^{* *}$ & $0.435^{* *}$ & $0.467 * *$ & $0.508^{* *}$ & 1 & $0.520^{* *}$ & $0.463^{* *}$ & $0.462^{* *}$ & $0.440^{* *}$ \\
\hline EWD & $0.638^{* *}$ & $0.545^{* *}$ & $0.551^{* *}$ & $0.714^{* *}$ & $0.679 * *$ & $0.590 * *$ & $0.520 * *$ & 1 & $0.598 * *$ & $0.540^{* *}$ & $0.621^{* *}$ \\
\hline HW & $0.722 *$ & $0.680^{* *}$ & $0.715^{* *}$ & $0.698^{* *}$ & $0.564 * *$ & $0.650 * *$ & $0.463 * *$ & $0.598 * *$ & 1 & $0.531 * *$ & $0.677 * *$ \\
\hline TL & $0.702^{* *}$ & $0.550^{* *}$ & $0.535^{* *}$ & $0.676^{* *}$ & $0.548 * *$ & $0.616^{* *}$ & $0.462 * *$ & $0.540 * *$ & $0.531 * *$ & 1 & $0.683^{* *}$ \\
\hline WT & $0.710^{* *}$ & $0.741^{* *}$ & $0.735^{* *}$ & $0.781^{* *}$ & $0.626^{* *}$ & $0.623 * *$ & $0.440 * *$ & $0.621 * *$ & $0.677 * *$ & $0.683^{* *}$ & 1 \\
\hline
\end{tabular}

Table 6: Correlations among measured body parameters in WAD goats at 2 years of age

\begin{tabular}{cccccccccccc}
\hline $\begin{array}{c}\text { Variable } \\
\text { s }\end{array}$ & $\mathbf{H ~ W}$ & $\mathbf{B L}$ & $\mathbf{H G}$ & $\mathbf{F L}$ & $\mathbf{H L}$ & $\mathbf{F A L}$ & $\mathbf{E L N}$ & EWD & HW & TL & WT \\
\hline HW & 1 & $0.472^{* *}$ & 0.026 & 0.262 & 0.108 & 0.336 & 0.007 & $0.428^{*}$ & 0.170 & 0.191 & $0.549^{* *}$ \\
BL & $0.472^{* *}$ & 1 & 0.141 & $0.359^{*}$ & $0.407^{*}$ & 0.188 & -0.119 & $0.515^{* *}$ & $0.501^{* *}$ & 0.251 & $0.489^{* *}$ \\
HG & 0.026 & 0.141 & 1 & -0.060 & 0.211 & 0.102 & 0.077 & $0.377^{*}$ & 0.202 & 0.259 & $0.537^{* *}$ \\
FL & 0.262 & $0.359^{*}$ & -0.060 & 1 & $0.495^{* *}$ & $0.484^{* *}$ & 0.194 & 0.199 & -0.011 & 0.077 & $0.453^{*}$ \\
HL & 0.108 & $0.407^{*}$ & 0.211 & $0.495^{* *}$ & 1 & 0.037 & -0.016 & 0.337 & -0.129 & 0.184 & 0.279 \\
FAL & 0.336 & 0.188 & 0.102 & $0.484^{* *}$ & 0.037 & 1 & 0.138 & -0.110 & 0.222 & -0.180 & $0.383 *$ \\
ELN & 0.007 & -0.119 & 0.077 & 0.194 & -0.016 & 0.138 & 1 & -0.135 & -0.080 & 0.151 & 0.110 \\
EWD & $0.428^{*}$ & $0.515^{* *}$ & $0.377^{*}$ & 0.199 & 0.337 & -0.110 & -0.135 & 1 & 0.080 & 0.337 & $0.392^{*}$ \\
HW & 0.170 & $0.501^{* *}$ & 0.202 & -0.011 & -0.129 & 0.222 & -0.080 & 0.080 & 1 & 0.088 & 0.316 \\
TL & 0.191 & 0.251 & 0.259 & 0.077 & 0.184 & -0.180 & 0.151 & 0.337 & 0.088 & 1 & $0.434^{*}$ \\
WT & $0.549^{* *}$ & $0.489^{* *}$ & $0.537^{* *}$ & $0.453^{*}$ & 0.279 & $0.383^{*}$ & 0.110 & $0.392^{*}$ & 0.316 & $0.434^{*}$ & 1 \\
\hline
\end{tabular}

ation is significant at the 0.01 leve

* Correlation is significant at the 0.05 level 
Effect of age, sex and local government area on linear body measurements of WAD goat

Table 7: Correlations among measured body parameters in WAD goats at 3 years of age

\begin{tabular}{cccccccccccc}
\hline $\begin{array}{c}\text { Variable } \\
\text { S }\end{array}$ & $\mathbf{H} \mathbf{W}$ & $\mathbf{B L}$ & $\mathbf{H G}$ & $\mathbf{F L}$ & $\mathbf{H L}$ & $\mathbf{F A L}$ & $\mathbf{E L N}$ & $\mathbf{E W D}$ & HW & TL & WT \\
\hline HW & 1 & $0.648^{* *}$ & $0.327^{*}$ & $0.611^{* *}$ & $0.638^{* *}$ & $0.601^{* *}$ & 0.310 & -0.225 & $0.608^{* *}$ & $0.689^{* *}$ & $0.719^{* *}$ \\
BL & $0.648^{* *}$ & 1 & $0.421^{* *}$ & $0.590^{* *}$ & $0.717^{* *}$ & $0.729^{* *}$ & $0.591^{* *}$ & 0.045 & $0.503^{* *}$ & $0.553^{* *}$ & $0.756^{* *}$ \\
HG & $0.327^{*}$ & $0.421^{* *}$ & 1 & 0.197 & 0.263 & $0.366^{*}$ & 0.048 & $0.340^{*}$ & $0.614^{* *}$ & 0.275 & $0.706^{* *}$ \\
FL & $0.611^{* *}$ & $0.590^{* *}$ & 0.197 & 1 & $0.840^{* *}$ & $0.508^{* *}$ & $0.475^{* *}$ & $-0.411^{*}$ & 0.306 & $0.475^{* *}$ & $0.458^{* *}$ \\
HL & $0.638^{* *}$ & $0.717^{* *}$ & 0.263 & $0.840^{* *}$ & 1 & $0.636^{* *}$ & $0.433^{* *}$ & $0.361^{*}$ & $0.364^{*}$ & $0.570^{* *}$ & $0.570^{* *}$ \\
FAL & $0.601^{* *}$ & $0.729^{* *}$ & $0.366^{*}$ & $0.508^{* *}$ & $0.636^{* *}$ & 1 & $0.362^{*}$ & 0.073 & $0.704^{*}$ & $0.542^{* *}$ & $0.685^{* *}$ \\
ELN & 0.310 & $0.591^{* *}$ & 0.048 & $0.475^{* *}$ & $0.433^{* *}$ & $0.362^{*}$ & 1 & 0.158 & $0.282^{*}$ & $0.383^{*}$ & $0.511^{* *}$ \\
EWD & -0.225 & 0.045 & $0.340^{*}$ & $0.411^{* *}$ & $-0.361^{* *}$ & 0.073 & 0.158 & 1 & $0.320^{*}$ & $-0.160^{* *}$ & 0.238 \\
HW & $0.608^{* *}$ & $0.503^{* *}$ & $0.614^{* *}$ & $0.306^{* *}$ & $0.364^{* *}$ & $0.704^{* *}$ & 0.282 & $0.320^{*}$ & 1 & $0.550^{* *}$ & $0.810^{* *}$ \\
TL & $0.689^{* *}$ & $0.553^{* *}$ & 0.275 & $0.475^{* *}$ & $0.570^{* *}$ & $0.542^{* *}$ & $0.383^{*}$ & -0.160 & $0.560^{* *}$ & 1 & $0.802^{* *}$ \\
WT & $0.719^{* *}$ & $0.756^{* *}$ & $0.706^{* *}$ & $0.458^{* *}$ & $0.570^{* *}$ & $0.685^{* *}$ & $0.511^{* *}$ & 0.238 & $0.810^{* *}$ & $0.802^{* *}$ & 1 \\
\hline
\end{tabular}

* Correlation is significant at the 0.05 level 
positive in age group 1 . In age group 2 it was significantly higher in fore limb length, face length ear width and tail length at $\mathrm{P}<0.05$ (Table 5 and 6). The correlation between body weights and all linear body measurements of age group 3 were positive and highly significant at $\mathrm{P}<0.01$ (Table 7). This also agreed with Barett et al. (1979) who reported that goat increased in body weights as the ages increases but nevertheless above 5 years of age, teeth erupt and they eat less which influences their body weight. It therefore agreed with the findings of Buvanendra et al (1981) that ewe weights did not show a similar trend with years and suggested that the decline in weight was not only nutritional but in breeding has contributed to the differences. It agreed with the submission of Fajemilehin and Salako (2008) who reported that age strongly influence body weight and body linear traits in WAD goats as there were consistent increases in all the traits studied as the animals aged.

\section{Conclusion}

Body weight and linear measurements are important traits in meat animals. Local government areas, towns, ages and sex had significant influences on body measurements at different ages and these should be considered in improvement programme to increase meat yield from WAD goat. Age significantly affected all body parameters measured in WAD goats, because the result showed an increase in the different live body measured as they matured. Sex significantly affected all body parameters of WAD goats measured and the value recorded in each parameter favoured the female WAD goats.

\section{References}

Adewunmi, O. O. and Badepade, E. G., 2008. Comparative weight and linear body measurement of adult lactating Yankasa and West African Dwarf ewes. Proceedings $33^{\text {rd }}$ Annual Conference of Nigerian Society for Animal Production (NSAP). Eds O. A. Adeyemi, A. O. Ogungbesan, A. O. Dada, O. O. Eniolorunda, H. A. Awojobi, D. B. Oke and J. A. Agunbiade. Held at Olabisi Onabanjo University, Yewa Campus Ayetoro. Pg. 14-16.

Adewunmi, O. O., Chineke, C. A., Alokan, J. A. and Onine, C., 2008. Weight and linear body measurements in Yankasa, West African Dwarf sheep and their crosses. Proceedings $33^{\text {rd }}$ Annual Conference of Nigerian Society for Animal Production (NSAP). Eds. O. A. Adeyemi, A. O. Ogungbesan, A. O. Dada, O. O. Eniolorunda, H. A. Awojobi, D. B. Oke and J. A. Agunbiade. Held at Olabisi Onabanjo University, Yewa Campus Ayetoro.Pg. 3-6.

Buvanendra, V., Adu, I. F. and Oyedola, B. A., 1981. Breed and environmental effects on lamb production in Nigeria. J.Agric. Sci. (Cambridge) 96: 9-15.

Campbell, J. R., Kenealy, D. M., Campbell, L. K., 2003. Breeds and life cycles of livestock and poultry. In Animal Science - The Biology, Care and Production of Domestic Animals. $4^{\text {th }}$ Edition Pg. 20-46.

Dalton, D. C., 1985. An introduction to practical Animal Breeding. $2^{\text {nd }}$ Edition. Gerald, W., 1994. The Tropical Agriculturalist. Macmillian Press Ltd. London. Pg54-57.

Fajemilehin Samuel, O. K. and Salako, A. E., 2008. Body measurement characteristics of the West African Dwarf (WAD) Goat in deciduous forest zone of South Western Nigeria. African Journal of Biotechnology Vol. 
Effect of age, sex and local government area on linear body measurements of WAD goat

7 (14), pp.2521-2526.

Ige, A. O., Akinlade, J. A., Ojedapo, L. O. Oladunjoye, I. O., Amad, S. R. and Animashaun, A. O., 2006. Effect of sex on interrelationship between body weight and linear body measurement of commercial broilers in a derived savannah environment of Nigeria. Proceedings $11^{\text {th }}$ Annual Conference of the Animal Science Association of Nigeria. 18-21 September 2006, Ibadan, Oyo State, Nigeria. Pp.231233.

McDonald, P., Edwards, R. and Green Halgh, J. F. D., 1981. Animal Nutrition. $3^{\text {rd }}$ Edition longman, London.
'Ngere, L. O., Adu, I. F. and Okubanjo, A. O., 1984. The indigenous goats of Nigeria. Animal genetic Resource Information. 3: 1-9.

Ogah, D. M., Musa, I. S. and Yusuf, N. D., 2013. Prediction of carcass weight from Body measurements in West African Dwarf goat using canonical correlation analysis. Eguptian Journal of sheep and goat sciences, Vol.8 (1), P: 75-79.

Stephen Sunday Acheneje Egena, Garba Hussemi, Timothy Silas and Tairu Ciroman Musa, 2010. Effect of sex on linear body measurements of Guinea Pig (Cavis porcellus) AU J. T. 14(1): 61-65.

Received: 02/04/13

Accepted: 05/09/13 